\title{
Ischemic Stroke in a Young Patient Heralding a Left Ventricular Noncompaction Cardiomyopathy
}

\author{
Fanny Lestienne ${ }^{a} \quad$ Chiara Bruno $^{a} \quad$ David Bertora $^{b} \quad$ Jeanne Benoit ${ }^{a}$ \\ Marie-Hélène Mahagne $^{a} \quad$ Laurent Suissa $^{a}$ \\ ${ }^{a}$ Stroke Unit, Pasteur II Hospital, Nice, France; ${ }^{b}$ Department of Cardiology, Pasteur II \\ Hospital, Nice, France
}

\section{Keywords}

Stroke $\cdot$ Young patients $\cdot$ Left ventricular noncompaction cardiomyopathy

\section{Abstract}

Strokes in young patients may be the clinical expression of many complex and extremely rare diseases. Uncommon causes constitute less than $5 \%$ of all strokes, but are present in $30 \%$ of strokes in young patients. We report the case of a young woman whose ischemic stroke led to the diagnosis of a rare embolic cardiomyopathy, left ventricular noncompaction cardiomyopathy, requiring a heart transplant.

\section{Introduction}

The etiology of strokes in young adults is a diagnostic challenge for clinicians. Uncommon causes constitute less than $5 \%$ of all strokes, but are present in $30 \%$ of strokes in young patients [1]. Among various causes, cardioembolic diseases are frequent ones to look for. We report the case of a young girl affected by an uncommon cardiomyopathy. 


\section{Case Reports in Neurology}

\section{Case Report}

A 23-year-old female was admitted to the emergency room because of abdominal pain with dyspnea. Last year, she presented a renal arterial embolism requiring a thrombectomy. The exploration of this renal infarction uncovered a dilated cardiomyopathy, initially presumed of viral origin, with heart failure requiring an implantable cardioverter defibrillator. When she was urgently readmitted, a computerized tomography (CT) scan revealed acute cholecystitis and an antibiotic therapy was begun. Given the cardiological history and the presence of dyspnea with elevated BNP, she was transferred to the cardiology ward. There, she presented a sudden right hemiparesis the next day with right hemianopsia and aphasia. Since implantable cardioverter defibrillators contraindicate magnetic resonance imaging (MRI), an emergency CT scan was performed and showed an acute left carotid T occlusion and a long-standing right internal carotid occlusion (Fig. 1). The patient was transferred to the stroke unit for systemic thrombolysis and thrombectomy. Throughout the procedure, middle cerebral artery recanalization was obtained, but a clot was dislodged in the ipsilateral anterior cerebral artery during the procedure (Fig. 2). After a short hospitalization in the intensive care unit for respiratory difficulties due to acute pulmonary edema, the patient was readdressed to the stroke unit where the neurological examination was normal.

Supposing a cardioembolic origin of the stroke, due to dilated cardiomyopathy, a heparin treatment was started. The remainder of the exhaustive etiological assessment was not contributory. No biological thrombophilia was found. Despite an effective anticoagulation (unfractionated heparin with anti-Xa activity in the target), the patient presented an embolic complication in the form of acute ischemia of the left lower limb which was successfully treated surgically. In addition, several episodes of sustained ventricular tachycardia requiring two internal electric shocks by the implantable defibrillator were recorded.

An echocardiographic evaluation showed a dilated left ventricular heart disease, high ventricular pressures with ejection fraction of $22 \%$, an overall severe hypokinesia, and the presence of left ventricular crypts with a typical aspect of "spongy myocardium." A diagnosis of left ventricular noncompaction cardiomyopathy (LVNC) was suspected and it was confirmed when the patient was transferred to the cardiology unit due to the results of the cardiac MRI and cardiac CT scan performed during her last year's hospitalization (Fig. 3). In the cardiology ward, 9 episodes of ventricular tachycardia causing syncope were recorded. The hospital stay was complicated by cardiogenic shock, requiring the patient's transfer to the transplant center where a heart transplant was done.

\section{Discussion}

Stroke in young patients may be the clinical expression of many complex and extremely rare diseases. Among the causes of stroke in young adults, it is important to consider cervical artery dissection, cardioembolic sources, hematological diseases, coagulopathies, metabolic diseases and other rare ones. LVNC is a rare congenital cardiomyopathy, which appears to represent an arrest in intrauterine endomyocardial morphogenesis [2]. It is characterized by prominent myocardial trabeculations and deep intertrabecular recesses in the left ventricular cavity [3]. In adults, the prevalence of LVNC is reported as 0.05\% [4]. In infants, the incidence is calculated as 0.80 per 100,000 individuals per year and in children as 0.12 per 100,000 individuals per year [5, 6]. Since the first publication in 1990 [7], LVNC has been associated with systemic thromboembolism, cardiac failure, cardiac arrhythmia or de- 


\section{Case Reports in Neurology}

pressed left ventricular systolic function [8]. Cardioembolism can manifest as stroke, transient ischemic attack, mesenteric infarction, myocardial infarction, renal infarction, or peripheral embolism [9]. They probably result from atrial arrhythmias, or impaired ejection fraction but thrombus formation within the noncompacted ventricle may also be relevant, supported by autopsy findings of thrombi located within deep intratrabecular recesses [7]. The frequency of thromboembolism is variable: $13-24 \%$ in adults $[5,10]$ and $0-38 \%$ in pediatric patients [9]. Atrial fibrillation or systolic dysfunction increased the risk for cardioembolic stroke or embolism [10]. No embolic events were reported in a pediatric population [11]; the rates of stroke or embolism seemed to depend on age, cardiovascular comorbidities, and the duration of follow-up [5]. LVNC is usually diagnosed using transthoracic echocardiography and more rarely by transesophageal echocardiography, ventriculography, cardiac CT or cardiac MRI [12]. The transthoracic echocardiography diagnostic criterion consists in calculating the ratio of noncompacted/compacted (N/C) endomyocardium layers, which has been defined as N/C $>2$ [13]. The intertrabecular recesses in the cardiac apex and/or the inferior and lateral left ventricular walls, which have been considered the most frequently affected segments [14], are prone to thrombus formation with a potential risk of secondary systemic embolism. This physiopathology of the thrombus constitution easily explains the poor efficacy of curative anticoagulation in this embolic dilated cardiomyopathy. There are no formal guidelines for the management of patients with LVNC; a therapeutic approach must be adapted for each patient [15].

\section{Conclusions}

We have reported the case of a young woman, with no significant cerebrovascular risk factor, in whom a stroke and a systemic embolism revealed the presence of a rare cardiomyopathy, leading to the need of a cardiac transplant. This case stresses the importance of an accurate diagnosis of stroke causes, as it can be associated with poor prognosis. LVNC, rare as it may be, should be considered as a possible cause of stroke in young adults. This pathology must be discussed in all young patients in whom severe nonischemic cardiomyopathy is associated with recurrences of cerebral and/or peripheral embolisms despite curative anticoagulation. This case also illustrates the usefulness of cardiac MRI in this rare diagnosis.

\section{Statement of Ethics}

The authors have no ethical conflicts to declare.

\section{Disclosure Statement}

The authors declare that there are no conflicts of interest and that no funding was received for this report. 
Lestienne et al.: Ischemic Stroke in a Young Patient Heralding a Left Ventricular Noncompaction Cardiomyopathy

\section{References}

1 Bogousslavsky J, Caplan L: Uncommon Causes of Stroke. Cambridge, Cambridge University Press, 2001

-2 Rigopoulos A, Rizos IK, Aggeli C, Kloufetos P, Papacharalampous X, Stefanadis C, Toutouzas P: Isolated left ventricular noncompaction: an unclassified cardiomyopathy with severe prognosis in adults. Cardiology 2002;98:25-32.

- Ikeda U, Minamisawa M, Koyama J: Isolated left ventricular non-compaction cardiomyopathy in adults. J Cardiol 2015;65:91-97.

-4 Ritter M, Oechslin E, Sütsch G, Attenhofer C, Schneider J, Jenni R: Isolated noncompaction of the myocardium in adults. Mayo Clin Proc 1997;72:26-31.

5 Oechslin E, Attenhofer C, Rojas J, Kaufmann P, Jenni R: Long-term follow-up of 34 adults with isolated left ventricular noncompaction: a distinct cardiomyopathy with poor prognosis. J Am Coll Cardiol 2000;36:493-500.

6 Arbustini E, Weidemann F, Hall Jl: Left ventricular noncompaction: a distinct cardiomyopathy or a trait shared by different cardiac diseases? J Am Coll Cardiol 2014;64:1840-1850.

7 Chin TK, Perloff JK, Williams RG, Jue K, Mohrmann R: Isolated noncompaction of left ventricular myocardium: a study of eight cases. Circulation 1990;82:507-513.

-8 Stollberger C, Winkler-Dworak M, Blazek G, Finsterer J: Cardiologic and neurologic findings in left ventricular hypertrabeculation/noncompaction relating to echocardiographic indication. Int J Cardiol 2007;119:28-32.

-9 Weiford BC, Subbarao VD, Mulhern, KM: Noncompaction of the ventricular myocardium. Circulation 2004;109:2965-2971.

-10 Stöllberger C, Blazek G, Dobias C, Hanafin A, Wegner C, Finsterer J: Frequency of stroke and embolism in left ventricular hypertrabeculation/noncompaction. Am J Cardiol 2011;108:1021-1023.

-11 Ichida F, Hamamichi Y, Miyawaki T, Ono Y, Kamiya T, Akagi T, Hamada H, Hirose O, Isobe T, Yamada K, Kurotobi S, Mito H, Miyake T, Murakami Y, Nishi T, Shinohara M, Seguchi M, Tashiro S, Tomimatsu H: Clinical features of isolated noncompaction of the ventricular myocardium. J Am Coll Cardiol 1999;34:233-240.

-12 Finsterer J, Stöllberger C, Towbin JA: Left ventricular noncompaction cardiomyopathy: cardiac, neuromuscular, and genetic factors. Nat Rev Cardiol 2017;14:224-237.

13 Murphy RT, Thaman R, Blanes JG, Ward D, Sevdalis E, Papra E: Natural history and familial characteristics of isolated left ventricular non-compaction. Eur Heart J 2005;26:187-192.

$\checkmark 14$ Domnitskaia TM, Sidorenko BA, Erokhina MG, Saidova MA: Non-compact left ventricular myocardium: current state of the problem. Kardiologiia 2006;46:63-68.

15 Gao X, Chen H, Wang P, Li H: Subendocardial perfusion deficits due to left ventricular non-compaction cardiomyopathy: a case report and literature review. Int J Clin Exp Med 2017;10:3809-3816. 


\section{Case Reports in Neurology}

\begin{tabular}{l|l}
\hline Case Rep Neurol 2017;9:204-209 \\
\hline DOI: 10.1159/000479957 & $\begin{array}{l}\text { @ 2017 The Author(s). Published by S. Karger AG, Basel } \\
\text { www.karger.com/crn }\end{array}$ \\
\hline
\end{tabular}

Lestienne et al.: Ischemic Stroke in a Young Patient Heralding a Left Ventricular Noncompaction Cardiomyopathy

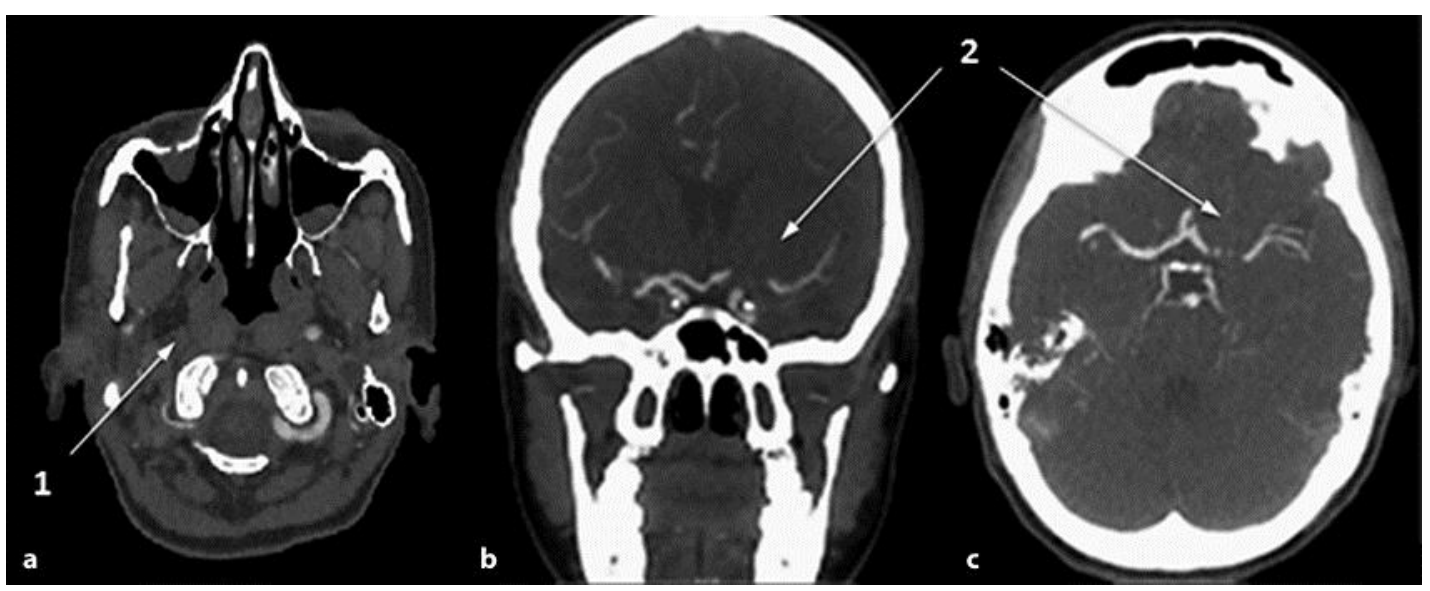

Fig. 1. a-c CT scan obtained on admission showing a long-standing right internal carotid occlusion (1) and an acute left carotid T occlusion (2).
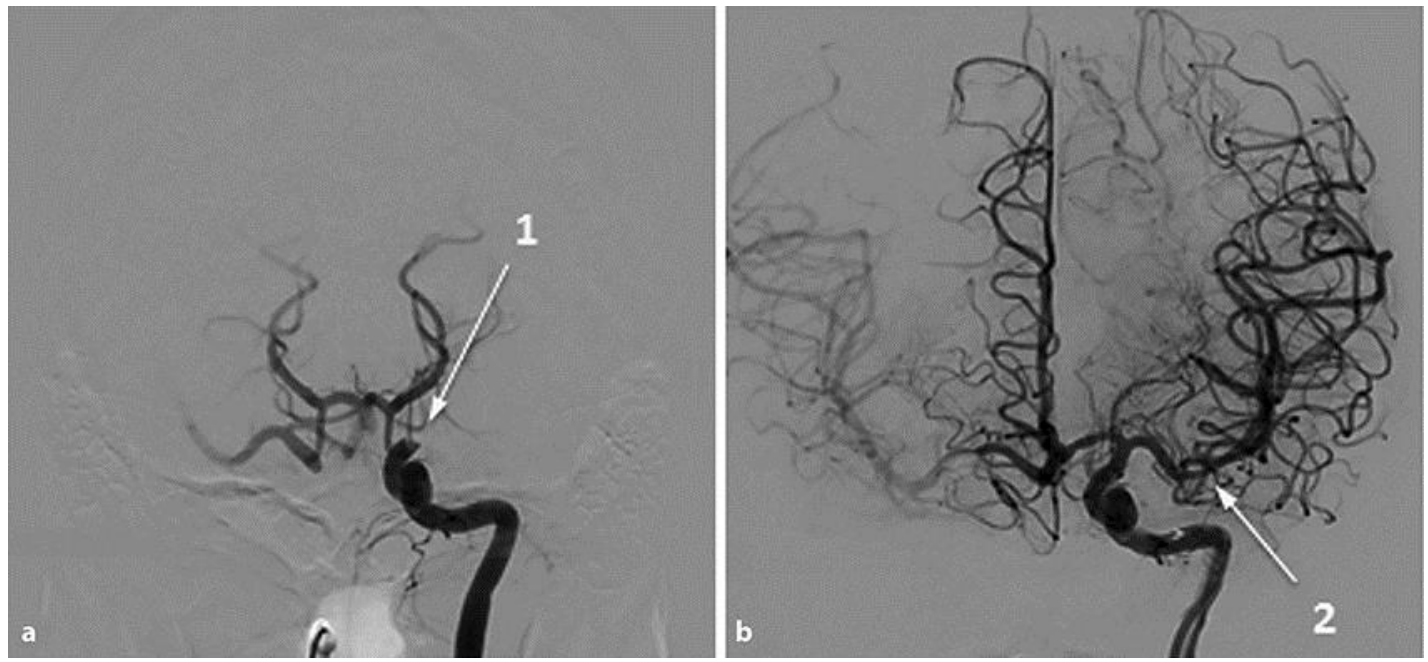

Fig. 2. Arteriography shows carotid $T$ occlusion $(a, 1)$, then the middle cerebral artery recanalization after thrombectomy (b, 2), but a clot was dislodged in the ipsilateral anterior cerebral artery during the procedure. 


\section{Case Reports in Neurology}
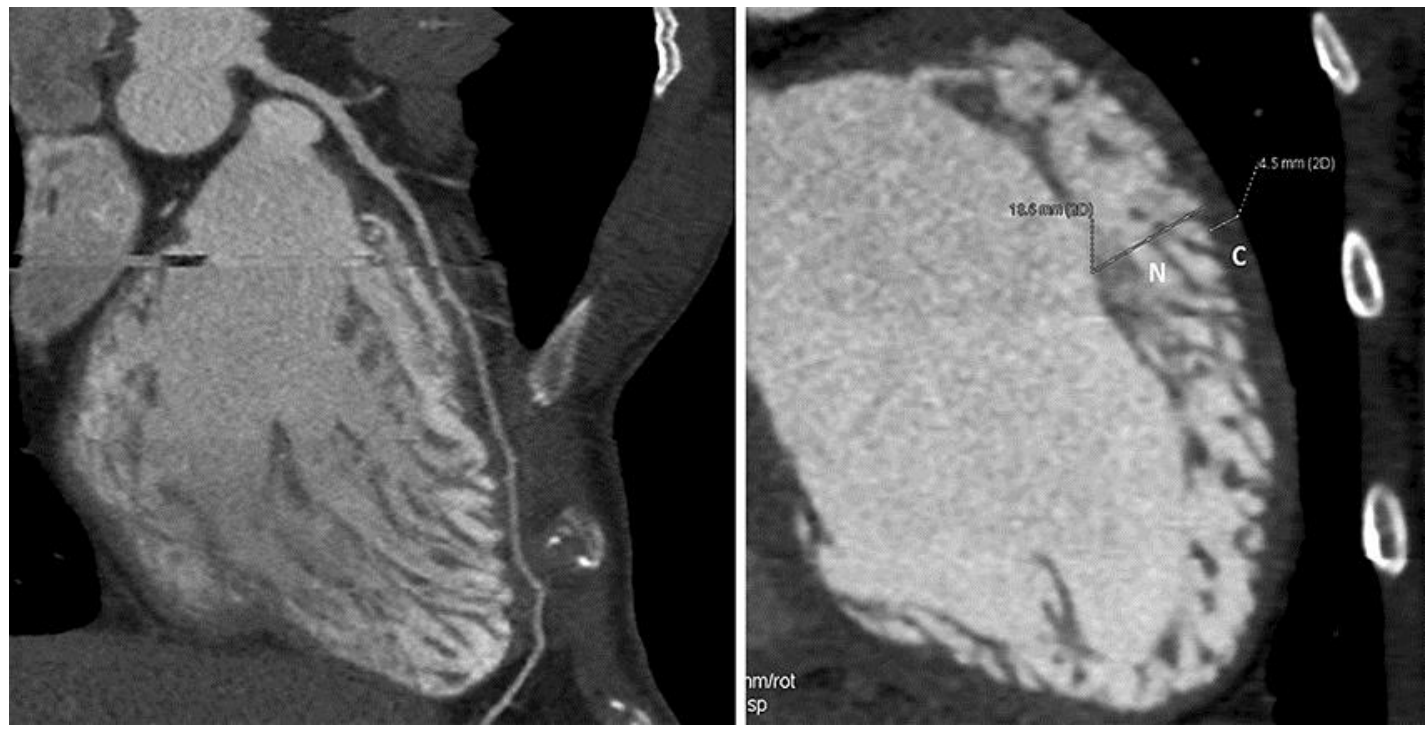

Fig. 3. Cardiac CT scan showed crypts and ratio of noncompacted (N)/compacted (C) endomyocardium greater than $2(n=18.6 \mathrm{~mm} ; \mathrm{C}=4.5 \mathrm{~mm})$. 